\begin{tabular}{l|l|l|l|l}
\hline Volume 2 & Issue 3 & December (2021) & DOI: 10.47540/ijsei.v2i3.389 & Page: $242-257$ \\
\hline
\end{tabular}

\title{
Analytical Profiling of Heavy Metals Contamination in soils, Dismantling Dust, and Rubber Samples in Karachi City Using AAS, WD-XRF, and SEM
} Technique

\author{
Abdul Rafeeq ${ }^{1}$, Syed Azhar Ali ${ }^{2}$, Asad Khan Tanoli ${ }^{3}$, Naseem Akhter ${ }^{4}$, Ghulam Raza ${ }^{5}$ \\ ${ }^{1,2,3}$ Department of Chemistry, University of Karachi, Pakistan \\ ${ }^{4,5}$ Peoples Steel Mills Ltd, Karachi, Pakistan
}

Corresponding Author: Abdul Rafeeq; Email: rafeequepak@gmail.com

A R T I C L E I N F O
Keywords: Baseline Data;
Contamination; EDX; Pak-EPA;
XRF.
Received $\quad: 08$ November 2021
$\begin{aligned} & \text { Revised } \quad: 13 \text { December } 2021 \\ & \text { Accepted } \quad: 14 \text { December } 2021\end{aligned}$

\begin{abstract}
A B S T R A C T
The concentrations of $\mathrm{Cd}, \mathrm{Cr}, \mathrm{Cu}, \mathrm{Fe}, \mathrm{Ni}, \mathrm{Pb}, \& \mathrm{Zn}$ were determined in the samples of soil, dismantling dust and rubber collected from the electronic waste dumping site of Shershah market and rubber from the Local market of Karachi city Pakistan. The city e-waste dumping and dismantling sites toxicity were not conducted before using modern techniques. The subsequent data of the heavy metals concentration were obtained using Atomic Absorption Spectrometry (AAS), Scanning Electron Microscopy (SEM), and Wavelength Dispersive- X-Ray Fluorescence Spectrometry (WD-XRF). $\mathrm{Cu}, \mathrm{Pb}$, and $\mathrm{Zn}$ were found as $133.17,104.53 \& 113.26$ in soil, whereas in dismantling dust $\mathrm{Pb} \& \mathrm{Zn}$ were remained 10.56 and $12.65 \mathrm{mg} / \mathrm{kg}$ similarly. The China toy particle analysis by SEM was resulting metallic trend as $\mathrm{Fe}>\mathrm{Zn}>\mathrm{Pb}>$ $\mathrm{Cd}>\mathrm{Ni}>\mathrm{Cr}$. The estimated data were compared with the levels allowed by the Pakistan Environmental Protection Agency (Pak-EPA) and the United States Environmental Protection Agency (USEPA). The maximum allowable limit for $\mathrm{Cd}$ is 3; $\mathrm{Cr}, \mathrm{Cd}$, and $\mathrm{Pb}$ are 100 for $\mathrm{Ni}$ is 50 and $300 \mathrm{mg} / \mathrm{kg}$ for $\mathrm{Zn}$. The presence of these heavy metals from e-waste dumping would become soon a significant reason to cause serious health problems for the nearby residents and as well as city too. It has been concluded that the dumping of e-waste is the major source of contamination of heavy metals in the studied media. It is recommended that the ewaste must be recycled formally to prevent the soil from being polluted.
\end{abstract}

\section{INTRODUCTION}

Discarded electronic and electrical appliances generate e-waste. These include computers, televisions, mobile phones, digital music recorders or players, refrigerators, washing machines, etc (Patil \& Ramakrishna, 2020) (Iqbal et al., 2015a). The presence of a mixture of various plastics and chemicals in e-waste can cause harmful impacts on humans and the environment owing to improper treatment of the waste (Singh, Duan, \& Tang, 2020) (Liu et al., 2018). The composition of the waste varies in products having different categories. The substances have been categorized into 'hazardous' and 'non-hazardous' substances and their number are more than 1000 (Perkins, Brune Drisse, Nxele, \& Sly, 2014). Among the hazardous substances in e-waste, there are heavy metals like Lead $(\mathrm{Pb})$,
Mercury (Hg), Arsenic (As), Cadmium (Cd), Selenium ( $\mathrm{Se}$ ), and Chromium ( $\mathrm{Cr}$ ). Globally, electronic waste is becoming a hastily growing issue (Forti, Baldé, Kuehr, \& Bel, 2020) (Kalamaras et al., 2021). About 40 to 50 million tons of e-waste is generated annually (Iqbal et al., 2015b)(Islam et al., 2020). The countries of Asia and Africa import e-waste where it is recycled and disposed off. As a result, it contaminates the soil, water, and air causing environmental issues (Faheem Gul Gilal, Syed Mir Muhammad Shah, Sultan Adeel, Rukhsana Gul Gilal, 2021). Therefore, workers and their children suffer from health problems (Bimir, 2020) (Leung, Anna O. W., et al., 2008).

The largest and oldest market of Scrap is in Shershah, Karachi, Pakistan. which is posing serious health and environmental issues to 
inhabitants. Almost 2 million people pass from this area daily (Iqbal et al., 2015a) (Rafeeq et al., 2020) (Umair, Anderberg, \& Potting, 2016) (Umair, Björklund, \& Petersen, 2015). Therefore, it is essential to determine the composition of the ewaste generated and dumped in the market. Analysis of the contaminants in e-waste dumpsites can be done by collecting the samples of dumpsite soil. The study of heavy metals in these significant environmental wastes can confer data in the atmosphere of the site (Iqbal et al., 2015a) (Mostafaii et al., 2021).

Workers in dismantling sites and their children are exposed to heavy metals through drinking of contaminated water, ingestion of contaminated food, inhalation of polluted air and dermal absorption. These metals can cause acute and chronic toxicity in the people, for example, the presence of $\mathrm{Pb}$ in the blood affects IQ of the children (Xu, Zeng, Boezen, \& Huo, 2015) (Olafisoye, Adefioye, \& Osibote, 2013). Studies have demonstrated that children and workers in ewaste dumpsites were contaminated with heavy metals and persistent organic substances (Liu et al., 2018) (Zeng, Xu, Boezen, \& Huo, 2016)(Imran, Haydar, Kim, Awan, \& Bhatti, 2017). Furthermore, surface water and plants have also been polluted with e-waste toxic substances (Olafisoye et al., 2013) (Michelle et al., 2016).

Literature reveals that Printed Circuit Boards (PCB) of $\mathrm{CPU}$ and monitor of computers are polluted with $\mathrm{Cu}$ and $\mathrm{Pb}$ and their concentrations were 50 folds more than the Toxicity Threshold Limit Concentration (TTLC) for the metals in electrical and electronic equipment in the developed countries (Singh, Duan, Ogunseitan, Li, \& Tang, 2019). Consequently, this equipment containing $\mathrm{Cu}$ and $\mathrm{Pb}$ are hazardous wastes. The disposal of the ewaste will cause health and environmental issues (Shaikh, Thomas, Zuhair, \& Magalini, 2020)(Li \& Achal, 2020). The purpose of the present work was to take initiative against e-waste contamination. This study will provide a quantitative analysis based on primary data to address the subsequent e-waste effects. The heavy metals in WEEE contaminated soil and groundwater were assessed using different Latest techniques like AAS, WD-XRF and SEM collected from the Shershah market area, Karachi.

\section{MATERIALS AND METHOdS Study Area}

The E-waste dumping site focused in this study is located $\left(24^{\circ} 52^{\prime} 52.7^{\prime \prime} \mathrm{N}\right.$ and $\left.66^{\circ} 59^{\prime} 40.0^{\prime \prime} \mathrm{E}\right)$ in Karachi, Sindh Provence in southeastern Pakistan. This dumpsite is mainly located to the second-hand goods supply market. Huge stuff of old material is available for purchase, considered as the largest reusable material market in Asia. E-waste recycling business is not older than 15-20 years in Pakistan (Iqbal et al., 2017). The specific recycling area and dumpsite were focused, where mostly the poor peoples and their children or hired children are involved in e-waste related activities. Since this area is closest to the seaport in Pakistan. Therefore, became the main receiver of e-waste. The main (50$60 \%$ ) recycling was carried out on this site. Another site was also selected for reference sampling where none of these types of activities were observed. Any difference in population, traffic density, lifestyle, rainfall, and socioeconomic status did not exist between the involved and controlled sites. The study was conducted from April to August in 20152018. A large no of soil samples was collected from the dumpsite and from control for reference (Umair et al., 2016) (Rafeeq, 2019).

\section{Collection of soil samples}

Soil samples were collected in April at various points of the e-waste dumpsite located (north to south) within the premises of Lyari expressway wall. 150-meter length of dumpsite was considered as an active site. The dumpsite was classified into A, B, C, D, and E points to determine the profile of the heavy metal in the soil. Designated point A covers about a fifty-meter wide (east to west) and thirty-meter long (north to south). The Lyari River cuts up the e-waste along with market waste dumping sites and opposite residential waste sites. The river let falls into the Arabian Sea.

Furthermore, the total area (150-meter long) was also divided into points $\mathrm{A}, \mathrm{B}, \mathrm{C}, \mathrm{D}$, and $\mathrm{E}$ to get comparative data. The marked areas were further subdivided such as A-1. A-2, A-3, A-4 and A-5. Uniform sampling was carried out from all areas. Stainless steel scale was used to drawn samples of burning ash from the areas keeping a depth up to $10 \mathrm{~cm}$. The five core samples were randomly collected and homogenized. A composite sample was taken as a representative sample of the whole soil point area. The composite sample was 
air-dried, crushed, and sieved through $2 \mathrm{~mm}$ sieve. The sample was stored in a polythene bag and labeled accordingly. A reference sample was also collected from the Nazimabad Cricket Playground 6.7 miles away from the dumpsite. In 2015-2018 (when the study was conducted for yearly monitoring) from the same dumping site, the surface and depth samples were collected by dividing the dumpsite into two parts $\mathrm{A} \& \mathrm{~B}$. The surface samples were marked as AS and BS while the depth samples were AD and BD (Rafeeq, 2019).

\section{Collection of e-waste surface dust samples}

The amount of dumping activity was investigated by the analysis of soil heavy metals. The heavy metals containing dust can become part of the human body through ingestion, inhalation, and dermal absorption (Ohajinwa, van Bodegom, Vijver, \& Peijnenburg, 2018). Weather conditions especially the dry season increase the prevalence of heavy metals in the dust (Juneng, Latif, \& Tangang, 2011) (Adaramodu, et el., 2012) (Olafisoye et al., 2013). Therefore, the samples of dust were analyzed from the dismantling sites. The sampling was carried out from three main units of the dismantling from streets no 08,09 , and 10 , where about $1000 \mathrm{~kg}$ per day e-waste is processed in each. The plastic brush and dustpans were used to collect fine particulates from 150-200 $\mathrm{g}$ dust by gentle sweeping motion. The auxiliaries were clean with tissue papers. Three composite samples of the dust were analyzed from the Shershah market and a control sample from Nazimabad playground. The samples were stored in plastic bags before the laboratory analysis. The samples were dried by keeping in the desiccators for $3 \mathrm{hrs}$, sieved using mesh (less than 2mm), and eventually homogenized. About $25 \mathrm{~g}$ of dust sample was grounded into a fine powder of 100 mesh size using a mini-mill (II), a ball mill before the chemical analysis (Rafeeq et al., 2020) (Rafeeq, 2019).

\section{Collection of Rubber samples}

The rubber sample was collected from the local market available in the form of china toys. Forty-four samples were selected and purchased from different local markets in Karachi. Samples were randomly picked from local market stalls; mall stores, bargain stores, roadside vendors, retail toy shops, and Imtiaz supermarket Gulshan e Iqbal Karachi. All samples were separated into two respective categories with code and labeled properly.

\section{Soil and Dust Sample Preparation for AAS} Analysis

The samples of soil and dust were digested with conc. $\mathrm{HNO}_{3}$ and extracted with concentrated $\mathrm{HCl}$ (Rafeeq et al., 2020) (Ehi-Eromosele C.O, Adaramodu A.A, Anake W.U, Ajanaku, \& EdoborOsoh, 2012). The wash glass and flask were washed with the deionized water and filtered into a $250 \mathrm{~mL}$ volumetric flask using a Whatman No. 42 filter paper, the filtrate was diluted up to the mark with the deionized water. A blank solution and a series of CRM sample were also prepared (Rafeeq, 2019) (Guo et al., 2010).

\section{Soil Samples Preparation for WD-XRF Analysis}

Soil samples were pounded by mixing with wax to get the homogenization by using a mini ball mill (a zirconium-based balls mill). The sample rotation in the machine was set clockwise and counters clockwise for 2 minutes at $240 \mathrm{rpm}$. Herzog press pellet machine was used to prepare the hard, flat, and smooth surface by applying $25 \mathrm{KN}$ force. Boric Acid was used as a binder to prevent the breaking or dispersion of samples during bombarding of high-intensity X-Rays (Ahmed, Hassan, Akhter, \& Mumtaz, 2019).

\section{Soil and Rubber Sample Preparation for SEM} Morphological Analysis

Soil (AS, AD, BS, and BD) and Rubber $(10 \times 10) \mathrm{mm}$ samples were bombarded with the Gold $(\mathrm{Au})$ particles. The Ion sputter coater machine was used to make the samples electrical conductor to avoid the building up charges during SEM analysis, which is necessary to analyze the sample with more sensitivity on SEM so that clear images should receive. Ion sputter coater machine is used to deposit metal coating that has a thickness of few nanometers, which is a very thin layer (Ahmed et al., 2019) (Hassan \& Naseem, 2020). 


\section{RESULTS AND DISCUSSION}

Heavy metal concentration $\mathrm{mg} / \mathrm{kg}$ of soil at E-waste dumpsite in (shershah) Karachi

Table 1. Average elemental concentrations in the soil surface $(0-10 \mathrm{~cm})(\mathrm{n}=15)$ using Atomic Absorption Spectrometer (ICE 3000 series) from (shershah dumpsite) Karachi (Rafeeq et al., 2020) (Rafeeq, 2019) (Ofudje, 2014).

\begin{tabular}{|c|l|l|l|l|l|l|}
\hline Ser \# & Sample ID & $\mathbf{Z n}(\mathbf{m g} / \mathbf{k g})$ & $\mathbf{N i}(\mathbf{m g} / \mathbf{k g})$ & $\mathbf{P b}(\mathbf{m g} / \mathbf{k g})$ & $\mathbf{C r}(\mathbf{m g} / \mathbf{k g})$ & $\mathbf{C u}(\mathbf{m g} / \mathbf{k g})$ \\
\hline 1 & $\mathrm{~A}$ & 125.41 & 4.61 & 112.11 & 2.14 & 80.38 \\
\hline 2 & $\mathrm{~B}$ & 84.73 & 6.31 & 75.78 & 1.44 & 373.87 \\
\hline 3 & $\mathrm{C}$ & 43.44 & 3.28 & 67.61 & 2.44 & 44.73 \\
\hline 4 & $\mathrm{D}$ & 21.36 & 1.90 & 44.12 & 1.24 & 17.69 \\
\hline 5 & $\mathrm{E}$ & 37.25 & 2.21 & 17.76 & 0.55 & 71.40 \\
\hline Mean & 62.436 & 3.662 & 63.474 & 1.562 & 117.614 \\
\hline Standard Deviation & 42.26 & 1.82 & 35.35 & 0.75 & 145.33 \\
\hline Median & 43.44 & 3.28 & 67.61 & 1.44 & 71.41 \\
\hline Min & 21.36 & 1.90 & 17.76 & 0.55 & 17.69 \\
\hline Max & 125.41 & 6.31 & 112.11 & 2.44 & 373.87 \\
\hline Control Sample & $0.015 \pm 0.001$ & $0.021 \pm 0.002$ & $\mathrm{ND} *$ & $0.011 \pm 0.005$ & $\mathrm{ND}$ \\
\hline USEPA & 300 & 50 & 100 & 100 & 100 \\
\hline Pak-EPA & 300 & 50 & 100 & 100 & 100 \\
\hline
\end{tabular}

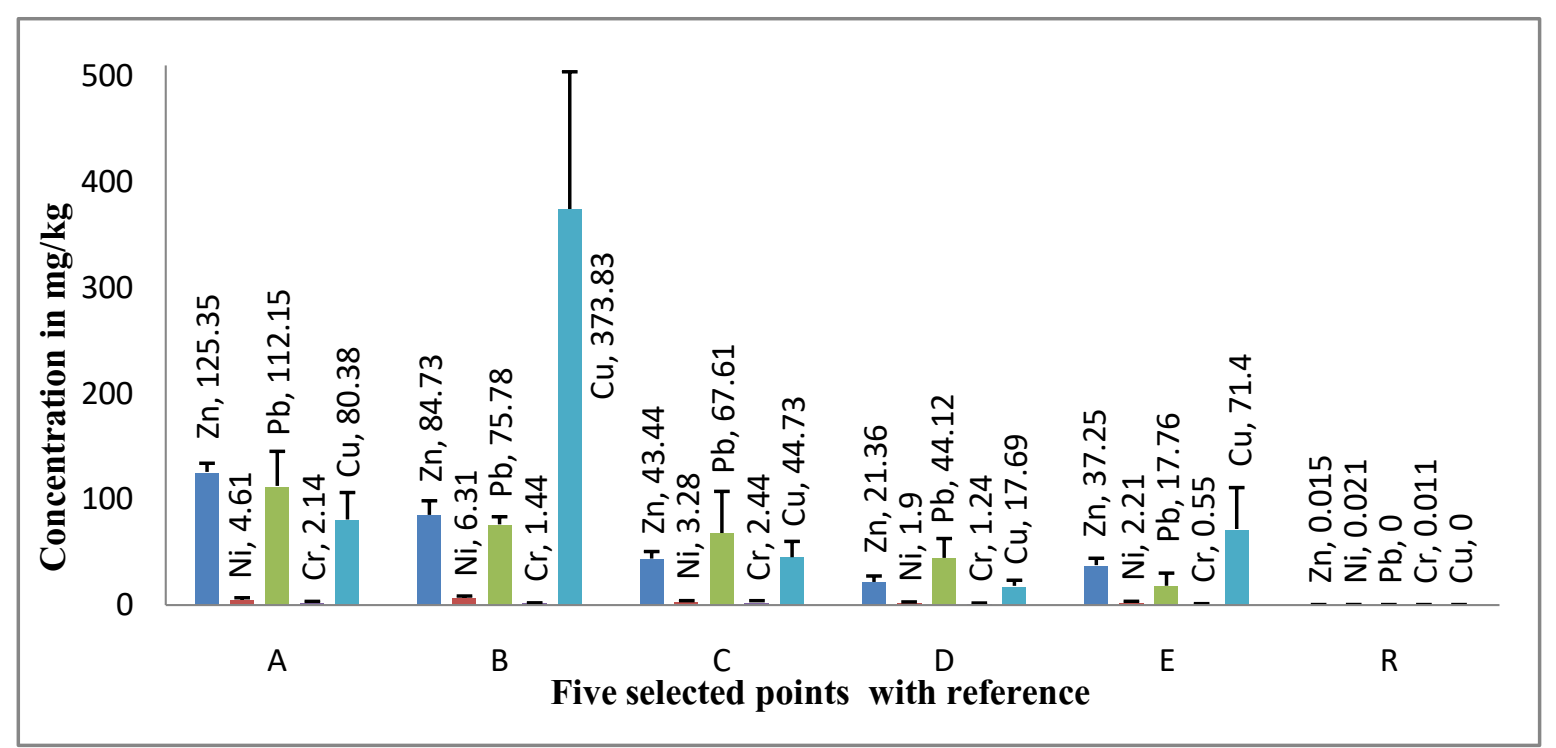

Figure 1. Total elemental concentrations $(\mathrm{mg} / \mathrm{kg})$ in the soil surface $(0-10 \mathrm{~cm})(\mathrm{n}=15)$.

Table 2. Maximum concentration ( $\mathrm{mg} / \mathrm{kg}$ ) of heavy metals in dry seasons from 2015-2018 (n=9) in the soil surface using Atomic Absorption Spectrometer (ICE 3000 series) (Rafeeq et al., 2020).

\begin{tabular}{|c|c|c|c|c|c|c|c|}
\hline Heavy Metals & $\mathbf{2 0 1 5}$ & $\mathbf{2 0 1 6}$ & $\mathbf{2 0 1 7}$ & $\mathbf{2 0 1 8}$ & USEPA & Pak-EPA & Remarks \\
\hline $\mathrm{Cd}$ & 0.58 & 0.53 & 0.42 & 0.52 & 3 & 3 & Low \\
\hline $\mathrm{Cr}$ & 2.91 & 1.95 & 1.10 & 1.23 & 100 & 100 & Low \\
\hline $\mathrm{Cu}$ & 332.41 & 136.71 & 113.37 & 133.17 & 100 & 100 & V high \\
\hline $\mathrm{Fe}$ & 5.90 & 5.90 & 3.86 & 3.98 & NA & NA & - \\
\hline $\mathrm{Ni}$ & 5.74 & 4.36 & 3.63 & 3.93 & 50 & 50 & Low \\
\hline $\mathrm{Pb}$ & 111.95 & 103.34 & 89.43 & 104.53 & 100 & 100 & Very high \\
\hline $\mathrm{Zn}$ & 125.35 & 121.71 & 93.46 & 113.26 & 300 & 300 & Relatively high \\
\hline
\end{tabular}


Table 3. Heavy metal concentration $(\mathrm{mg} / \mathrm{kg}$ ) of dust samples collected from dismantling sites. ( $\mathrm{n}=9)$ using Atomic Absorption Spectrometer (ICE 3000 series) (Rafeeq, 2019).

\begin{tabular}{|c|c|c|c|c|}
\hline $\begin{array}{l}\text { Heavy } \\
\text { Metal }\end{array}$ & $\begin{array}{l}\text { Dismantling dust } \\
\text { street } 08\end{array}$ & $\begin{array}{l}\text { Dismantling dust } \\
\text { street } 9\end{array}$ & $\begin{array}{l}\text { Dismantling dust } \\
\text { street } 10\end{array}$ & $\begin{array}{l}\text { Controlled dust } \\
\text { sample }\end{array}$ \\
\hline $\mathrm{Cd}$ & $0.046 \pm 0.001$ & $0.069 \pm 0.001$ & $0.055 \pm 0.001$ & ND \\
\hline $\mathrm{Cr}$ & $0.16 \pm 0.02$ & $0.23 \pm 0.032$ & $0.18 \pm 0.014$ & $0.0011 \pm 0.0003$ \\
\hline $\mathrm{Cu}$ & $0.76 \pm 0.03$ & $0.88 \pm 0.09$ & $1.03 \pm 0.11$ & $0.012 \pm 0.004$ \\
\hline $\mathrm{Fe}$ & $1.21 \pm 0.01$ & $1.11 \pm 0.08$ & $1.36 \pm 0.04$ & $1.15 \pm 0.03$ \\
\hline $\mathrm{Ni}$ & $0.065 \pm 0.03$ & $0.088 \pm 0.05$ & $0.072 \pm 0.06$ & $0.021 \pm 0.002$ \\
\hline $\mathrm{Pb}$ & $9.43 \pm 0.01$ & $10.56 \pm 0.04$ & $9.45 \pm 0.03$ & ND \\
\hline $\mathrm{Zn}$ & $10.41 \pm 0.03$ & $12.65 \pm 0.03$ & $9.69 \pm 0.08$ & $0.02 \pm 0.005$ \\
\hline $\mathrm{pH}$ & $7.89 \pm 0.09$ & $7.85 \pm 0.07$ & $7.88 \pm 0.06$ & $7.56 \pm 0.06$ \\
\hline \multirow[t]{2}{*}{ 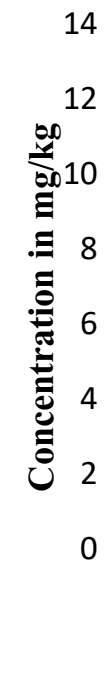 } & 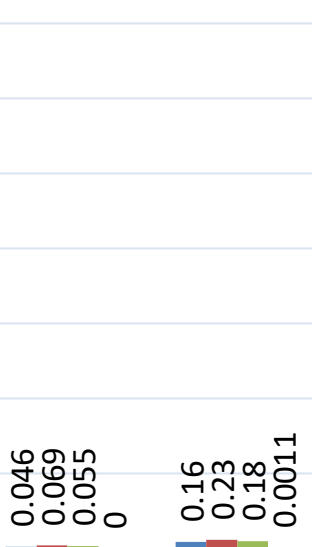 & 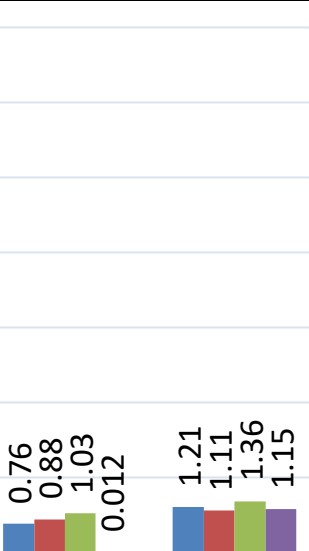 & 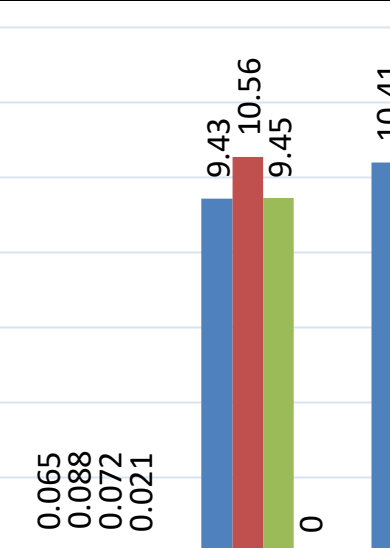 & 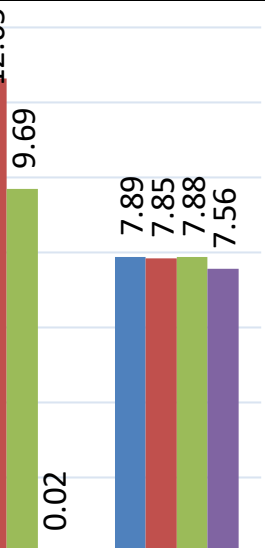 \\
\hline & \multicolumn{2}{|c|}{$\begin{array}{l}\mathrm{Cd} \quad \mathrm{Cr} \quad \mathrm{Cu} \\
\text { Dismantling dust street } 08 \\
\text { Dismantling dust street } 10\end{array}$} & $\begin{array}{l}\mathrm{Ni} \quad \stackrel{\mathrm{Pb}}{ } \\
\text { Dismantling dust street } \mathrm{S} \\
\text { Dismantling dust control }\end{array}$ & In sample \\
\hline
\end{tabular}

Figure 2. Heavy metals concentration $(\mathrm{mg} / \mathrm{kg})$ of dust sample collected from dismantling sites (April 2017).

Table 4. Heavy Metallic Elemental Composition $(\mathrm{mg} / \mathrm{kg})$ in pressed pallet for surface and depth soil in 2017Analyzed on WD-XRF Analyzer (Axios Model by PANalytical).

\begin{tabular}{|c|c|c|c|c|c|}
\hline Heavy Metal & AS & AD & BS & BD & USEPA \\
\hline $\mathrm{Cd}$ & ND & ND & ND & ND & 3 \\
\hline $\mathrm{Cr}$ & 0.82 & 0.13 & 1.16 & 0.57 & 100 \\
\hline $\mathrm{Cu}$ & 52.37 & 7.18 & 122.86 & 42.53 & 100 \\
\hline $\mathrm{Fe}$ & 3.15 & 1.16 & 4.57 & 2.97 & NA \\
\hline $\mathrm{Ni}$ & 3.07 & 0.56 & 3.27 & 2.34 & 100 \\
\hline $\mathrm{Pb}$ & 94.79 & 23.42 & 70.45 & 20.47 & 100 \\
\hline $\mathrm{Zn}$ & 101.85 & 14.27 & 83.16 & 9.36 & 300 \\
\hline
\end{tabular}


Results of Scanning Electron Microscope (SEM) analysis of surface soil in 2019

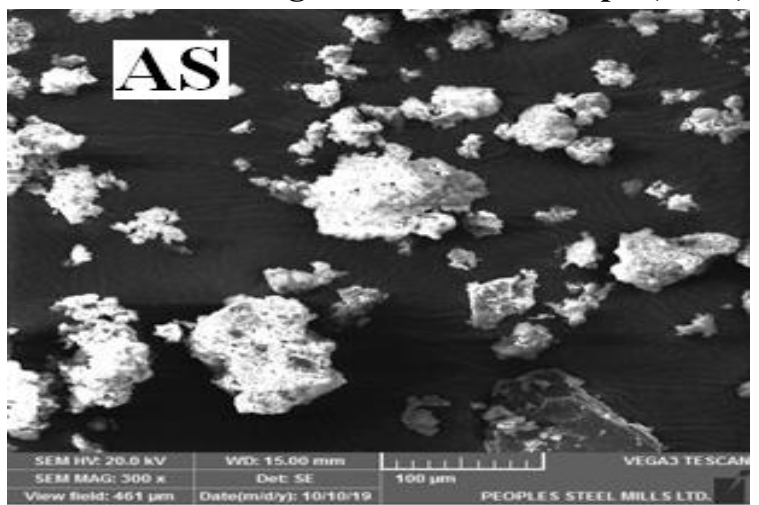

Figure 3. Scanning Electron Microscopic image of soil particles of side A surface (shershah dumpsite), Karachi
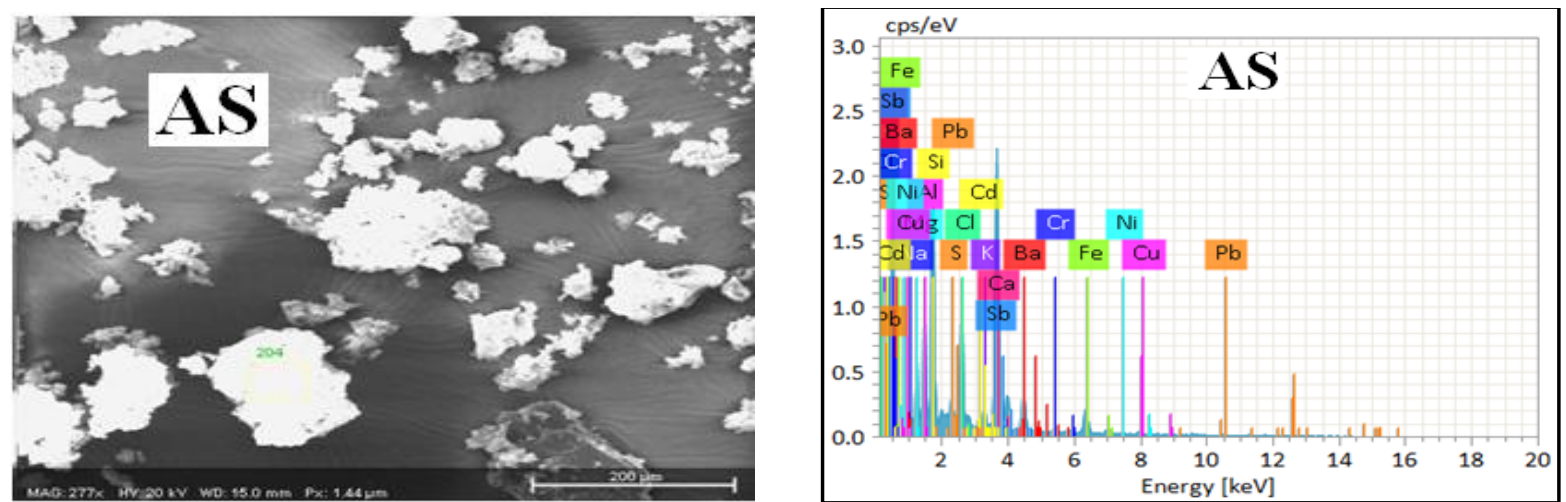

Figure 4. SEM image of soil particles of AS at magnification of 277X with Graph of SEM-EDX (Energy Dispersive X-Rays) analysis of elements.
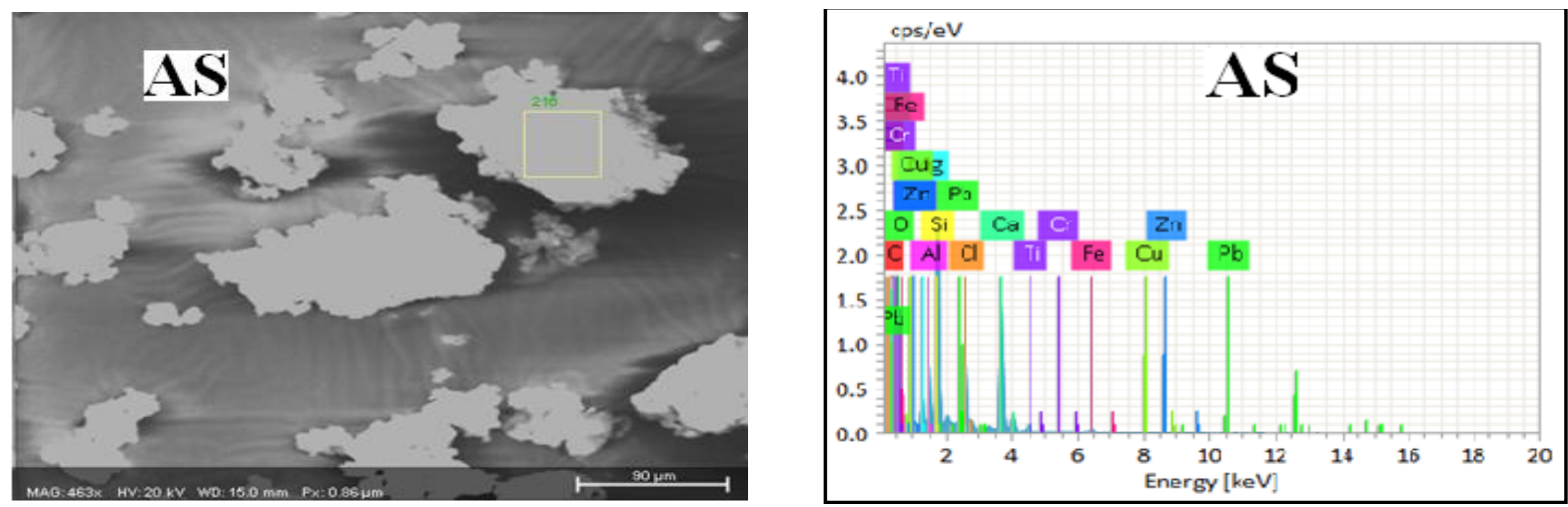

Figure 5. AS sample particle SEM image at a magnification of 463X with Graph of SEM-EDX analysis.

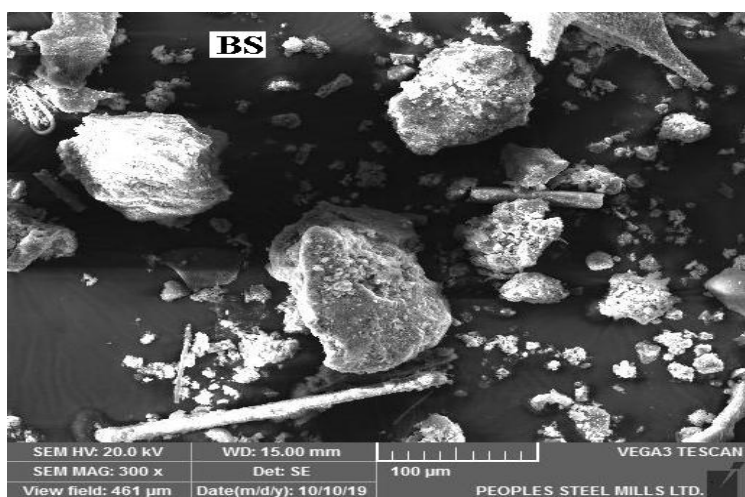

Figure 6. SEM image of soil particles of BS (shershah dumpsite), Karachi. 

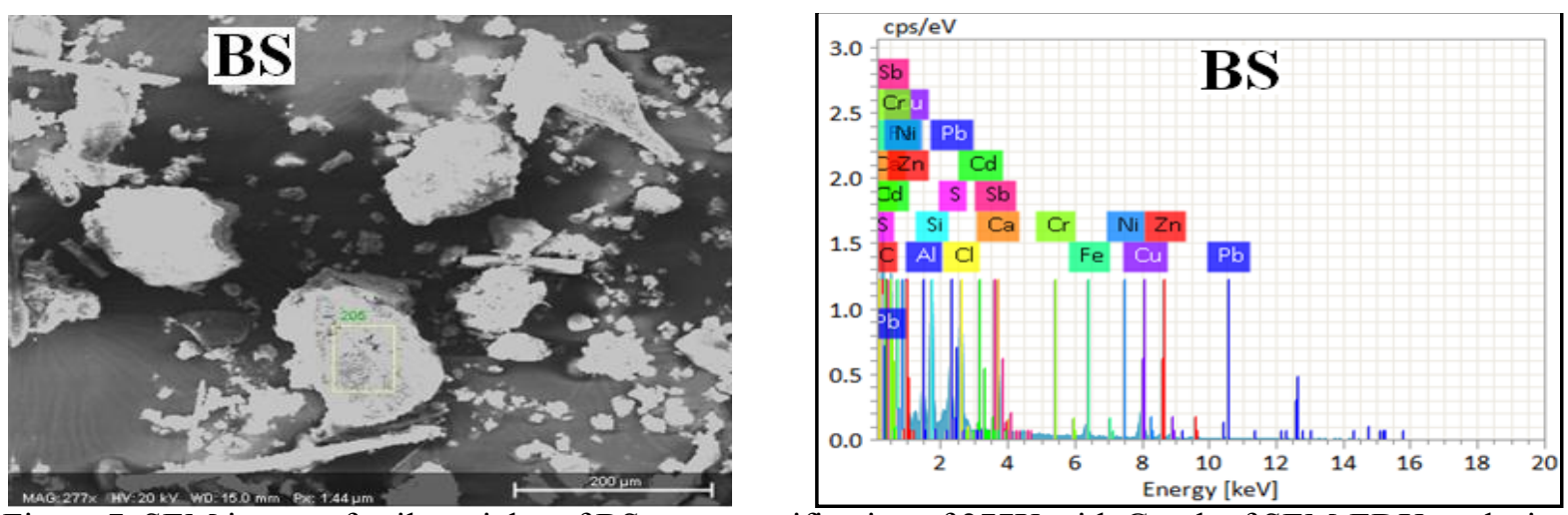

Figure 7. SEM image of soil particles of BS at a magnification of 277X with Graph of SEM-EDX analysis.

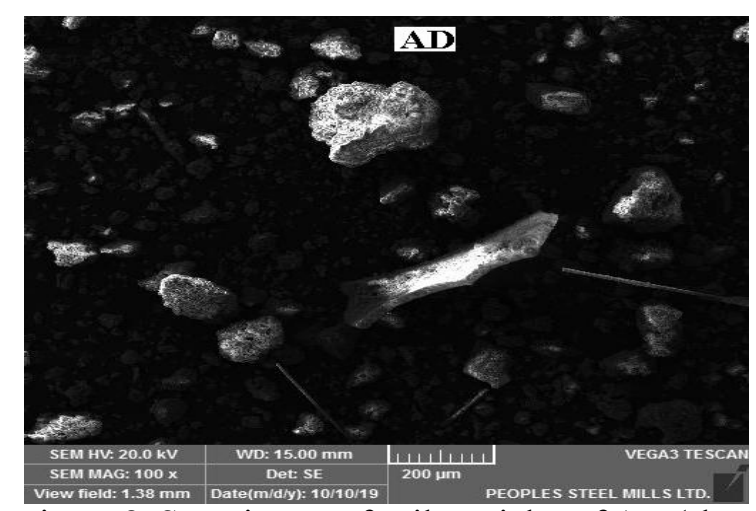

Figure 8. SEM image of soil particles of AD (shershah dumpsite), Karachi
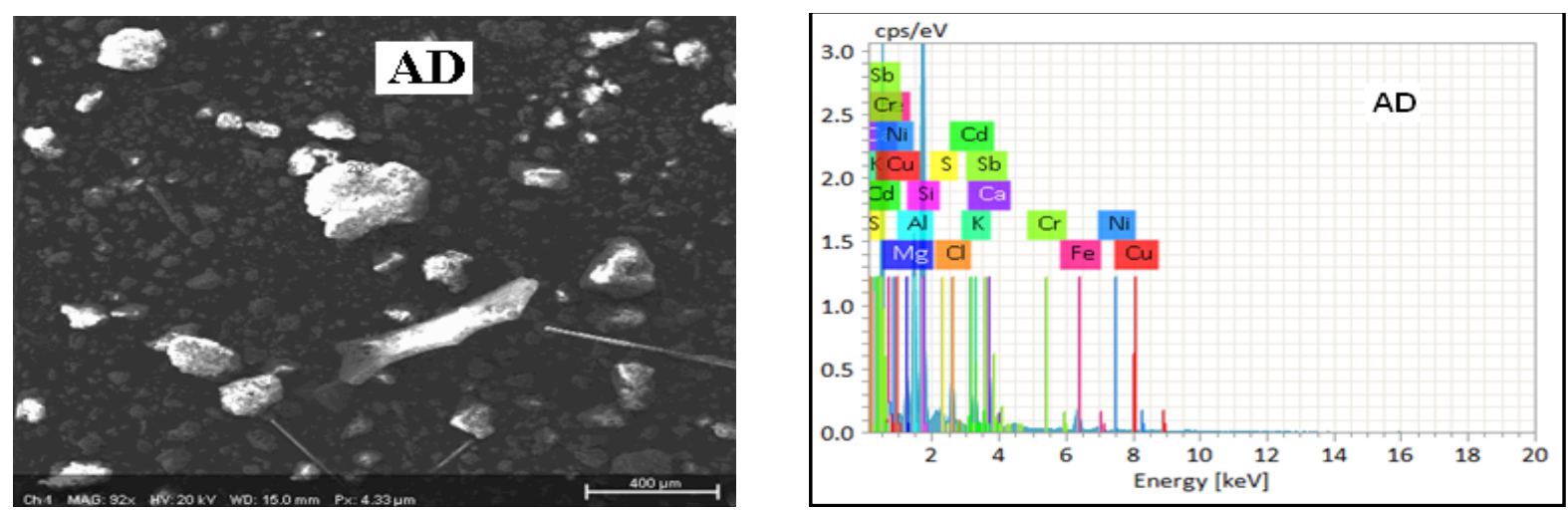

Figure 9. SEM image of soil particles of AD at a magnification of 92X with Graph of SEM-EDX analysis.
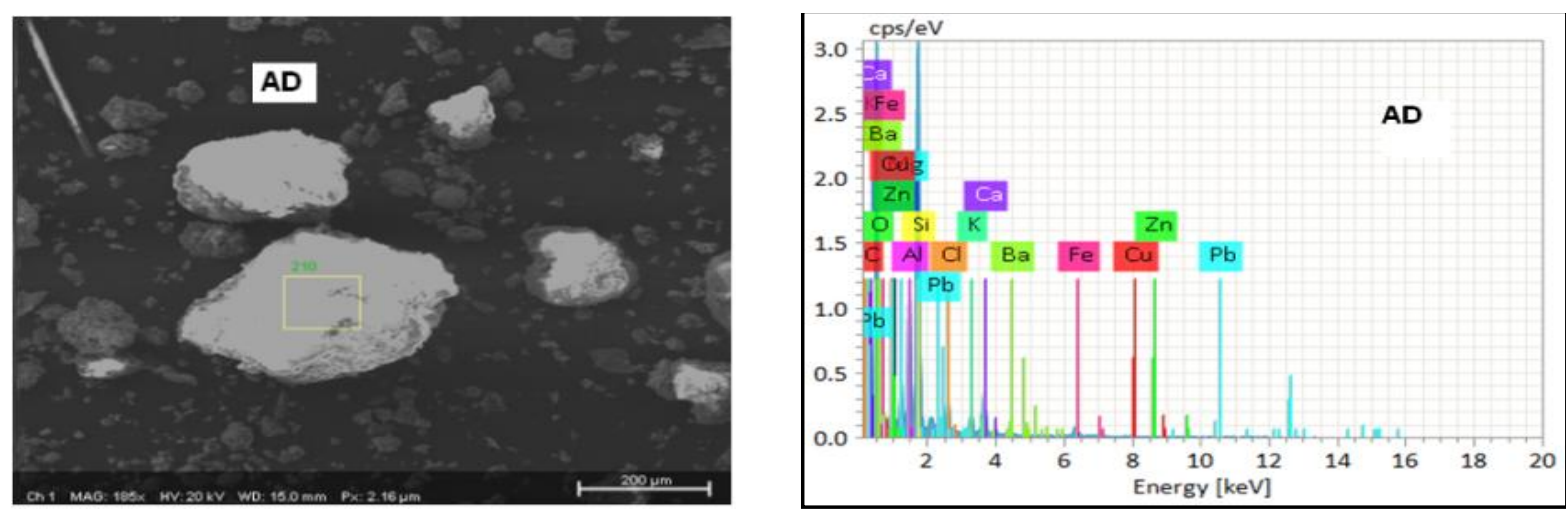

Figure 10. AD sample particle SEM image at a magnification of 185X with Graph of SEM-EDX analysis. 


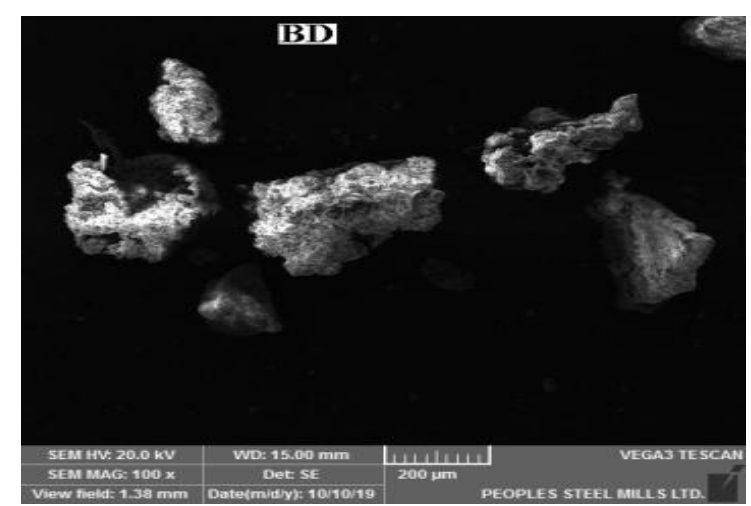

Figure 11. SEM (TASCAN, VEGA 3 SERIES) image at 100X of soil particles of Side B depth (shershah dumpsite), Karachi
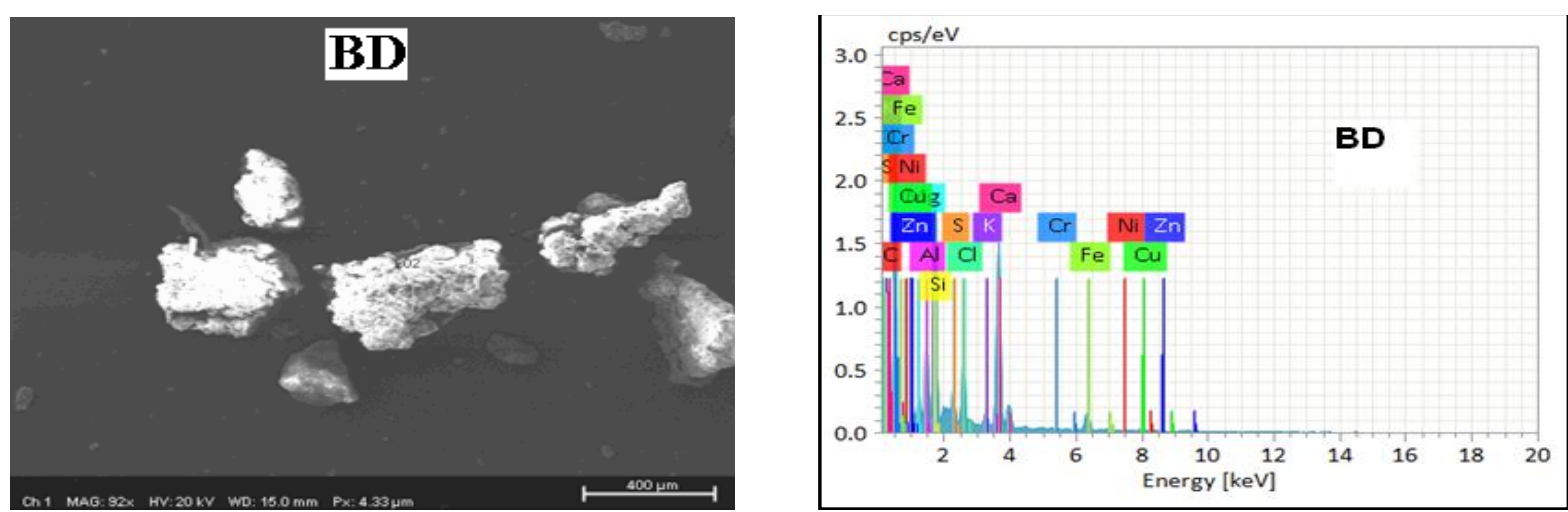

Figure 12. SEM image of soil particles of BD at a magnification of 92X with Graph of SEM-EDX analysis.
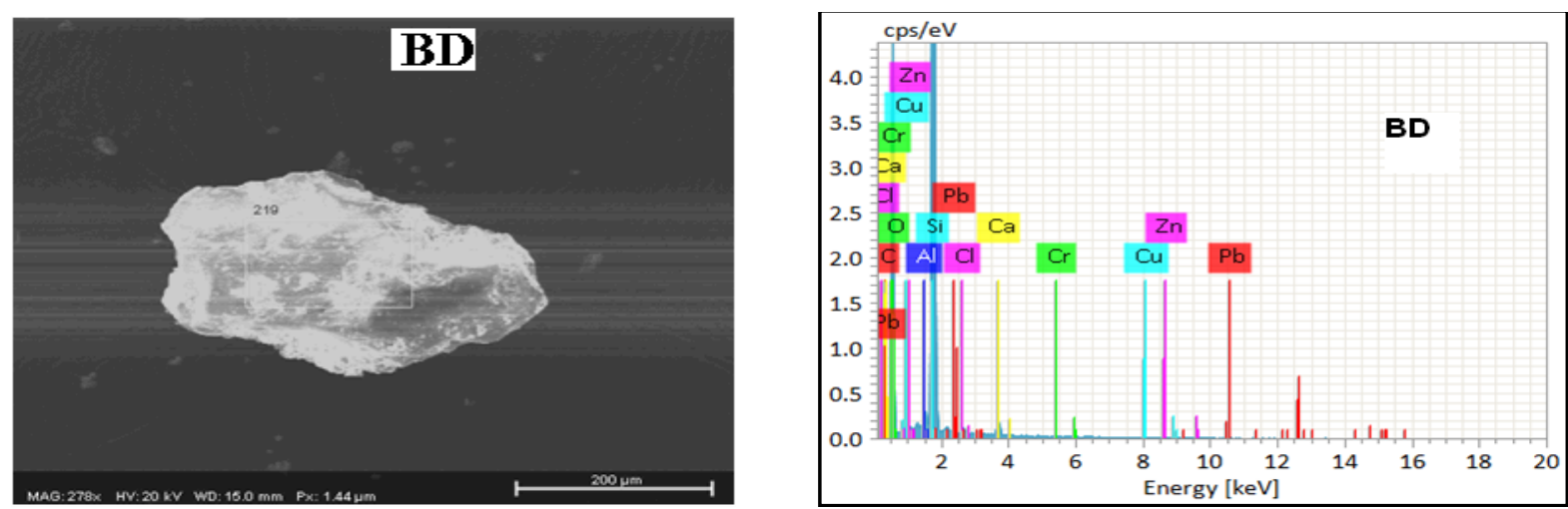

Figure 13. SEM image of soil particles of side B depth at a magnification of 278X with Graph of SEMEDX analysis

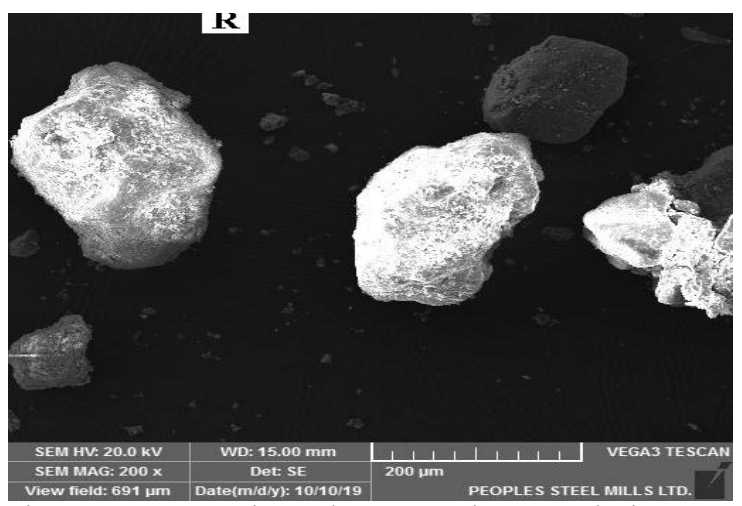

Figure 14. Scanning Electron Microscopic image of soil particle of reference (R) sample at 200X, Karachi 

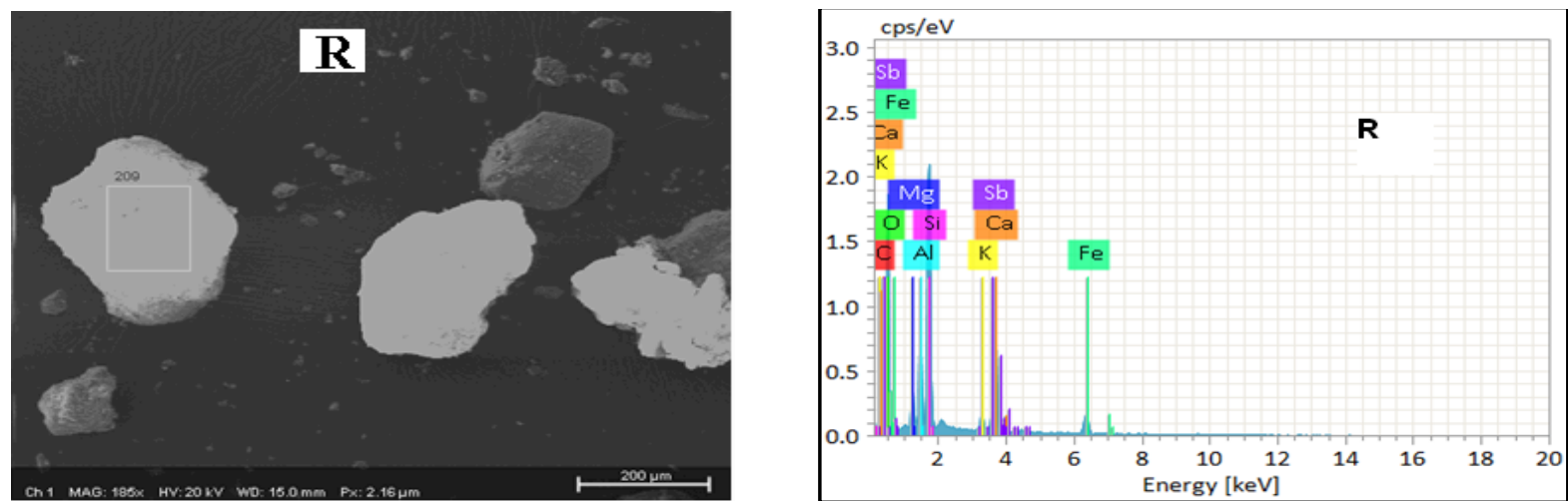

Figure 15. SEM image of soil particles of reference sample $\mathrm{R}$ at a magnification of $185 \mathrm{X}$ with Graph of SEM-EDX analysis of selected particle in image $\mathrm{R}$

\section{Results of Scanning Electron Microscope (SEM) analysis of Rubber sample collected in 2018}
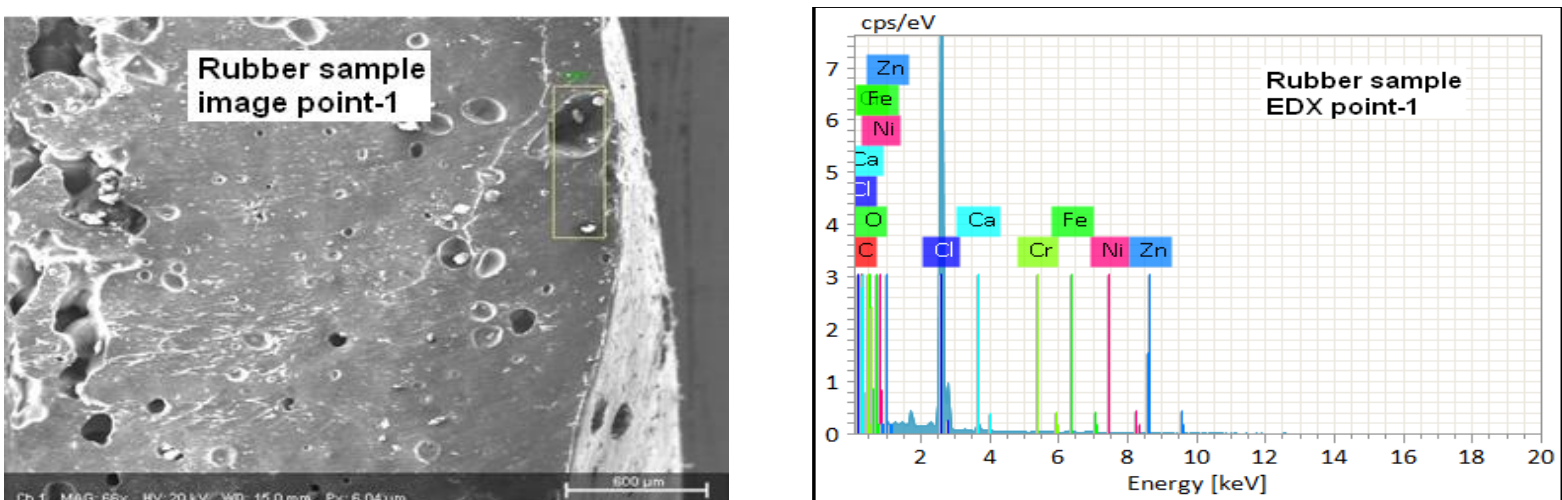

Figure 16. SEM image of rubber sample particle at a magnification of 66X with Graph of SEM-EDX analysis of elements of a selected particle in rubber sample image-point 1
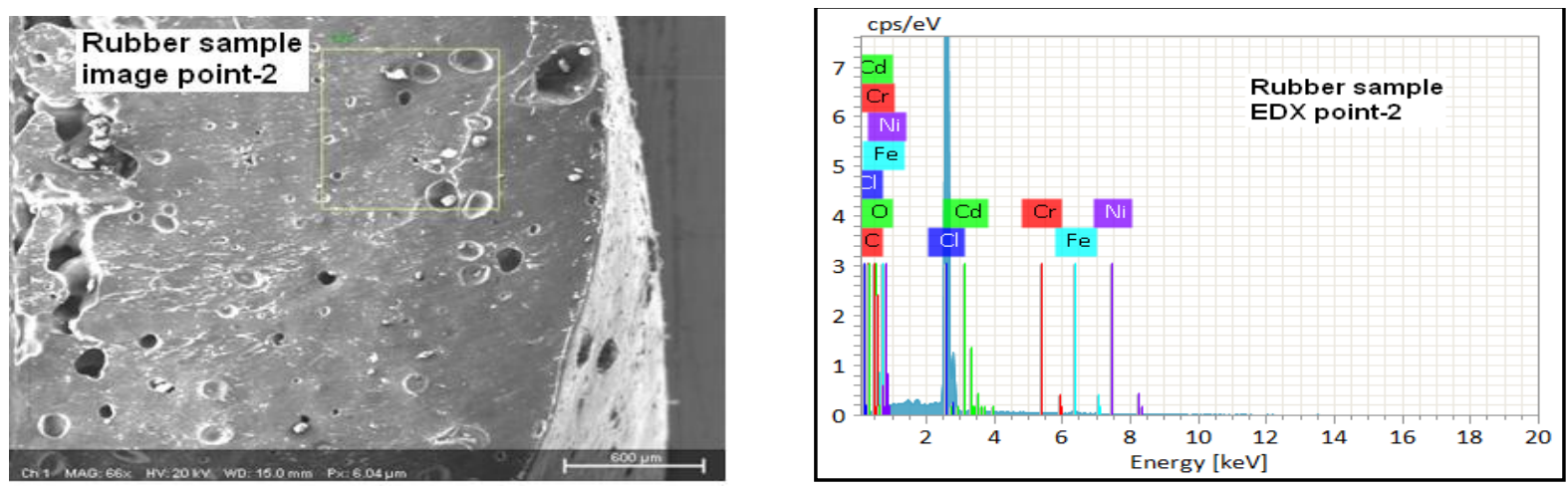

Figure 17. SEM image of rubber sample particle at a magnification of 66X with Graph of SEM-EDX analysis of elements of a selected particle in rubber sample image-point 2
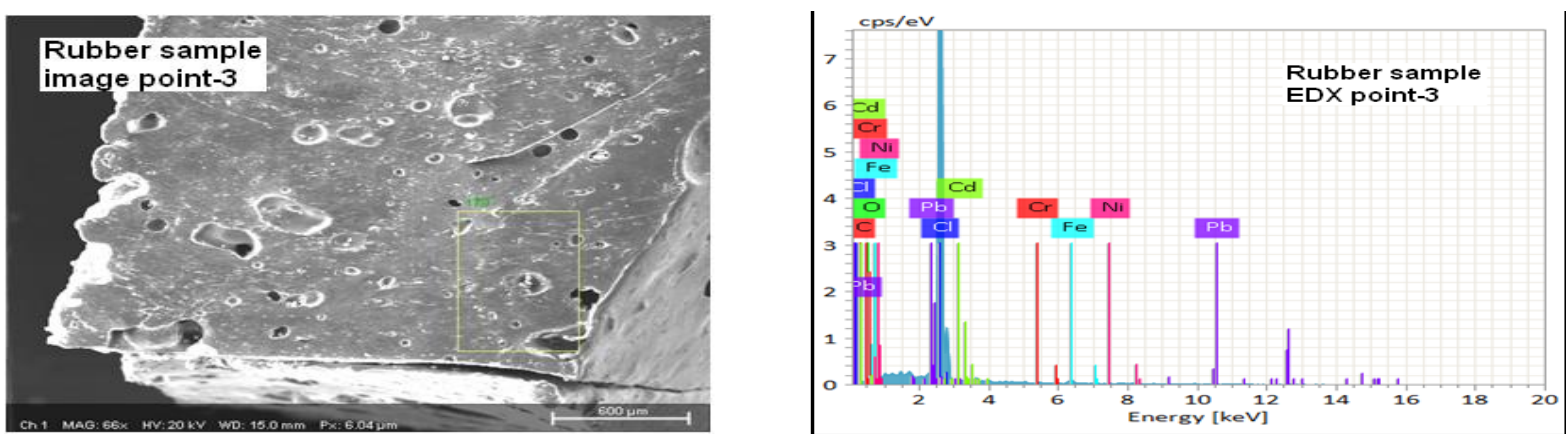

Figure 18. SEM image of rubber sample particle at magnification of 66X with Graph of SEM-EDX analysis of elements of selected particle in rubber sample image-point 3 


\section{Results of Scanning Electron Microscope (SEM) analysis of depth soil and control sample marked as $\mathbf{R}$ in 2019}

Table 5. The chemical concentration of heavy metals present in selected particle surface and depth soil at different magnification by SEM-EDX

\begin{tabular}{|c|c|c|c|c|c|c|c|c|c|}
\hline $\begin{array}{c}\text { Serial } \\
\#\end{array}$ & $\begin{array}{l}\text { Sample } \\
\text { ID }\end{array}$ & Elements & $\begin{array}{c}\mathrm{Cd} \\
(\mathrm{mg} / \mathrm{kg})\end{array}$ & $\begin{array}{c}\mathrm{Cr} \\
(\mathrm{mg} / \mathrm{kg})\end{array}$ & $\begin{array}{c}\mathrm{Cu} \\
(\mathrm{mg} / \mathrm{kg})\end{array}$ & $\begin{array}{c}\mathrm{Fe} \\
(\mathrm{mg} / \mathrm{kg})\end{array}$ & $\begin{array}{c}\mathrm{Ni} \\
(\mathrm{mg} / \mathrm{kg})\end{array}$ & $\begin{array}{c}\mathrm{Pb} \\
(\mathrm{mg} / \mathrm{kg})\end{array}$ & $\begin{array}{c}\mathrm{Zn} \\
(\mathrm{mg} / \mathrm{kg})\end{array}$ \\
\hline \multirow{3}{*}{01} & \multirow{3}{*}{ 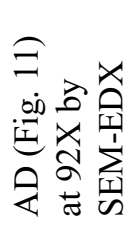 } & Concentration & 2809 & 376 & 3138 & 47126 & 1055 & - & - \\
\hline & & $\begin{array}{c}\text { \%Abs. error } \\
\text { (1 sigma) }\end{array}$ & 612 & 134 & 790 & 2507 & 541 & - & - \\
\hline & & $\begin{array}{l}\text { \%Rel. error } \\
\text { (1 sigma) }\end{array}$ & 21.8 & 35.8 & 25.2 & 5.3 & 51.3 & - & - \\
\hline \multirow{3}{*}{02} & \multirow{3}{*}{ 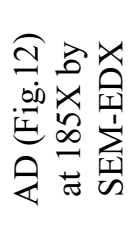 } & Concentration & - & - & 1905 & 14559 & - & 3310 & 902 \\
\hline & & $\begin{array}{c}\% \text { Abs. error } \\
\text { (1 sigma) }\end{array}$ & - & - & 611 & 1175 & - & 1193 & 497 \\
\hline & & $\begin{array}{c}\text { \% Rel. error } \\
\text { (1 sigma) }\end{array}$ & - & - & 32.1 & 8.1 & - & 36.1 & 55.2 \\
\hline \multirow{3}{*}{03} & \multirow{3}{*}{ 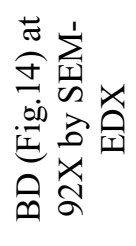 } & Concentration & - & 519 & 597 & 22113 & 78 & - & 1606 \\
\hline & & $\begin{array}{c}\text { \% Abs. error } \\
\text { (1 sigma) }\end{array}$ & - & 370 & 418 & 1345 & 52.8 & - & 556 \\
\hline & & $\begin{array}{l}\text { \% Rel. error } \\
\text { (1 sigma) }\end{array}$ & - & 71.2 & 70.1 & 6.1 & 67.5 & - & 34.7 \\
\hline \multirow{3}{*}{04} & \multirow{3}{*}{ 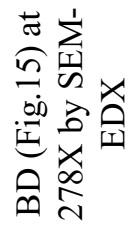 } & Concentration & - & 555 & 2100 & - & - & 4239 & 367 \\
\hline & & $\begin{array}{c}\% \text { Abs. error } \\
\text { (1 sigma) }\end{array}$ & - & 389 & 656 & - & - & 1377 & 164 \\
\hline & & $\begin{array}{l}\text { \% Rel. error } \\
\text { (1 sigma) }\end{array}$ & - & 70.2 & 31.2 & - & - & 32.5 & 44.8 \\
\hline \multirow{3}{*}{05} & \multirow{3}{*}{ 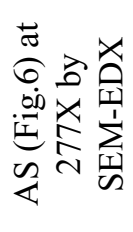 } & Concentration & 5842 & 10832 & 7167 & 45688 & 778 & 8747 & - \\
\hline & & $\begin{array}{c}\text { \% Abs. error } \\
\text { (1 sigma) }\end{array}$ & 766 & 1043 & 1142 & 2406 & 497 & 2276 & - \\
\hline & & $\begin{array}{l}\text { \% Rel. error } \\
\text { (1 sigma) }\end{array}$ & 13.1 & 9.6 & 15.9 & 5.3 & 63.9 & 13.1 & - \\
\hline \multirow{3}{*}{06} & \multirow{3}{*}{ 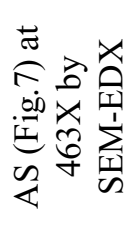 } & Concentration & - & 563 & 2927 & 15011 & - & 4043 & - \\
\hline & & $\begin{array}{l}\text { \% Abs. error } \\
\text { (1 sigma) }\end{array}$ & - & 376 & 169 & 1165 & - & 1228 & - \\
\hline & & $\begin{array}{l}\text { \% Rel. error } \\
\text { (1 sigma) }\end{array}$ & - & 66.8 & 34 & 7.76 & - & 30.4 & - \\
\hline \multirow{3}{*}{07} & \multirow{3}{*}{ 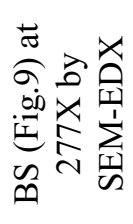 } & Concentration & 2103 & 866 & 55574 & 14260 & 2290 & 1207 & 3760 \\
\hline & & $\begin{array}{c}\text { \% Abs. error } \\
\text { (1 sigma) }\end{array}$ & 466 & 399 & 2610 & 1068 & 573 & 716 & 725 \\
\hline & & $\begin{array}{l}\text { \% Rel. error } \\
\text { (1 sigma) }\end{array}$ & 22.1 & 46.1 & 4.7 & 7.5 & 25.1 & 59.3 & 19.3 \\
\hline \multirow{3}{*}{08} & \multirow{3}{*}{ 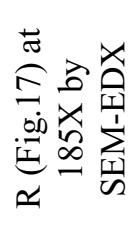 } & Concentration & - & - & - & 35218 & - & - & - \\
\hline & & $\begin{array}{c}\text { \% Abs. error } \\
\text { (1 sigma) }\end{array}$ & - & - & - & 1790 & - & - & - \\
\hline & & $\begin{array}{c}\text { \% Rel. error } \\
\text { (1 sigma) }\end{array}$ & - & - & - & 5.1 & - & - & - \\
\hline
\end{tabular}


Table 6 . The chemical concentration of heavy metals present in selected rubber particle at 66X by SEMEDX

\begin{tabular}{|c|c|c|c|c|c|c|c|}
\hline $\begin{array}{c}\text { Sample } \\
\text { point }\end{array}$ & Element & $\begin{array}{c}\mathrm{Cd} \\
(\mathrm{mg} / \mathrm{kg})\end{array}$ & $\begin{array}{c}\mathrm{Cr} \\
(\mathrm{mg} / \mathrm{kg})\end{array}$ & $\begin{array}{c}\mathrm{Fe} \\
(\mathrm{mg} / \mathrm{kg})\end{array}$ & $\begin{array}{c}\mathrm{Ni} \\
(\mathrm{mg} / \mathrm{kg})\end{array}$ & $\begin{array}{c}\mathrm{Pb} \\
(\mathrm{mg} / \mathrm{kg})\end{array}$ & $\begin{array}{c}\mathrm{Zn} \\
(\mathrm{mg} / \mathrm{kg})\end{array}$ \\
\hline \multirow{3}{*}{ 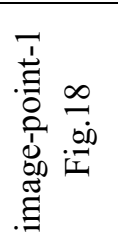 } & Concentration & - & 544 & 4735 & 186 & - & 1298 \\
\hline & $\begin{array}{c}\text { \% Abs. error } \\
\text { (1 sigma) }\end{array}$ & - & 369 & 672 & 78.9 & - & 506 \\
\hline & $\begin{array}{c}\text { \% Rel. error } \\
\text { (1 sigma) }\end{array}$ & - & 67.8 & 14.2 & 42.3 & - & 38.9 \\
\hline \multirow{3}{*}{ 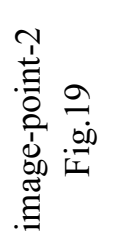 } & Concentration & 755 & 322 & 5279 & 580 & - & - \\
\hline & $\begin{array}{c}\text { \% Abs. error } \\
\text { (1 sigma) }\end{array}$ & 395 & 95 & 719 & 404 & - & - \\
\hline & $\begin{array}{c}\text { \% Rel. error } \\
\text { (1 sigma) }\end{array}$ & 52.3 & 29.4 & 13.6 & 69.6 & - & - \\
\hline \multirow{3}{*}{ 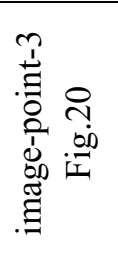 } & Concentration & 47 & 430 & 7275 & 407 & 983 & - \\
\hline & $\begin{array}{c}\text { \% Abs. error } \\
\text { (1 sigma) }\end{array}$ & 33 & 113 & 797 & 127 & 732 & - \\
\hline & $\begin{array}{c}\text { \% Rel. error } \\
\text { (1 sigma) }\end{array}$ & 70.1 & 26.4 & 10.9 & 31.2 & 74.5 & - \\
\hline
\end{tabular}

Table 7. Comparison of heavy metallic concentration $(\mathrm{mg} / \mathrm{kg}$ ) in soil, dismantling dust, and Rubber (China toy) samples analyzed on AAS (ICE-3000series), WD-XRF Analyzed (Axios Model), and SEM using maximum and minimum values of respected analysis.

\begin{tabular}{|c|c|c|c|c|c|c|c|c|}
\hline S\# & Sample ID & Cd & $\mathrm{Cr}$ & $\mathbf{C u}$ & $\mathrm{Fe}$ & $\mathbf{N i}$ & $\mathbf{P b}$ & $\mathbf{Z n}$ \\
\hline 01 & $\begin{array}{l}\text { Between profiling } \\
\text { sample A-E by AAS. }\end{array}$ & $\mathrm{NC}$ & $\begin{array}{l}2.44 \\
\text { to } \\
0.55\end{array}$ & $\begin{array}{l}373.87 \\
\text { to } 17.69\end{array}$ & $\mathrm{NC}$ & $\begin{array}{l}6.31 \\
\text { to } \\
1.90\end{array}$ & $\begin{array}{l}112.15 \\
\text { to } \\
17.76\end{array}$ & $\begin{array}{l}125.35 \\
\text { to } \\
26.36\end{array}$ \\
\hline 02 & $\begin{array}{l}\text { Between four years } \\
\text { monitoring dumpsite } \\
\text { samples by AAS. }\end{array}$ & $\begin{array}{l}0.58 \\
\text { to } \\
0.42\end{array}$ & $\begin{array}{l}2.91 \\
\text { to } \\
1.10\end{array}$ & $\begin{array}{l}332.41 \\
\text { to } \\
113.37\end{array}$ & $\begin{array}{l}5.90 \\
\text { to } \\
3.86\end{array}$ & $\begin{array}{l}5.74 \\
\text { to } \\
3.63\end{array}$ & $\begin{array}{l}111.95 \\
\text { to } \\
89.43\end{array}$ & $\begin{array}{l}125.35 \\
\text { to } \\
93.46\end{array}$ \\
\hline 03 & $\begin{array}{l}\text { Dismantling dust } \\
\text { samples by AAS. }\end{array}$ & $\begin{array}{l}0.069 \\
\text { to } \\
0.046\end{array}$ & $\begin{array}{l}0.23 \\
\text { to } \\
0.16\end{array}$ & $\begin{array}{l}1.03 \text { to } \\
0.76\end{array}$ & $\begin{array}{l}1.36 \\
\text { to } \\
1.11\end{array}$ & $\begin{array}{l}0.088 \\
\text { to } \\
0.065\end{array}$ & $\begin{array}{l}10.56 \\
\text { to } \\
9.43\end{array}$ & $\begin{array}{l}12.65 \\
\text { to } 9.69\end{array}$ \\
\hline 04 & $\begin{array}{l}\text { Surface and depth soil } \\
\text { samples by WD-XRF } \\
\text { Analyzer. }\end{array}$ & ND & $\begin{array}{l}0.82 \\
\text { to } \\
0.13\end{array}$ & $\begin{array}{l}122.86 \\
\text { to } 7.18\end{array}$ & $\begin{array}{l}4.57 \\
\text { to } \\
1.16\end{array}$ & $\begin{array}{l}3.27 \\
\text { to } \\
0.56\end{array}$ & $\begin{array}{l}94.79 \\
\text { to } \\
20.47\end{array}$ & $\begin{array}{l}101.85 \\
\text { to } 9.36\end{array}$ \\
\hline 05 & $\begin{array}{l}\text { Surface soil by SEM- } \\
\text { EDX. }\end{array}$ & $\begin{array}{l}5842 \\
\text { to } \\
2103\end{array}$ & $\begin{array}{l}10832 \\
\text { to } \\
563\end{array}$ & $\begin{array}{l}55574 \text { to } \\
2927\end{array}$ & $\begin{array}{l}45688 \\
\text { to } \\
14260\end{array}$ & $\begin{array}{l}2290 \\
\text { to } \\
778\end{array}$ & $\begin{array}{l}8747 \\
\text { to } \\
1207\end{array}$ & $\begin{array}{l}3760 \text { to } \\
0\end{array}$ \\
\hline 06 & $\begin{array}{l}\text { Depth soil by SEM- } \\
\text { EDX. }\end{array}$ & $\begin{array}{l}2809 \\
\text { to } \\
0\end{array}$ & $\begin{array}{l}555 \\
\text { to } \\
376\end{array}$ & $\begin{array}{l}3138 \text { to } \\
597\end{array}$ & $\begin{array}{l}47127 \\
\text { to } \\
14559\end{array}$ & $\begin{array}{l}1055 \\
\text { to } \\
78\end{array}$ & $\begin{array}{l}4239 \\
\text { to } \\
3310\end{array}$ & $\begin{array}{l}1606 \text { to } \\
367\end{array}$ \\
\hline 07 & $\begin{array}{l}\text { Rubber sample (China } \\
\text { toy) by SEM-EDX. }\end{array}$ & $\begin{array}{l}755 \\
\text { to } \\
47\end{array}$ & $\begin{array}{l}544 \\
\text { to } \\
322\end{array}$ & ND & $\begin{array}{l}7275 \\
\text { to } \\
4735\end{array}$ & $\begin{array}{l}580 \\
\text { to } \\
186\end{array}$ & $\begin{array}{l}983 \\
\text { to } \\
0\end{array}$ & $\begin{array}{l}1298 \text { to } \\
825\end{array}$ \\
\hline
\end{tabular}

Table 1 shows the heavy metal elemental 700B \& ICE 3000 series) of $\mathrm{Zn}, \mathrm{Ni}, \mathrm{Pb}, \mathrm{Cr}$, and $\mathrm{Cu}$ concentration by AAS spectrometer (A analyst in the soil of electronic waste dumpsite (shershah) 
Karachi. During 2015-2018 analysis was carried out to assess the soil contamination in the designated distribution for the whole dumpsite. The results are summarized only for maximum concentration comparatively in table 2 detailed results were published (Rafeeq et al., 2020). The higher concentrations within the samples were found to be $508 \mathrm{mg} / \mathrm{kg}$ of $\mathrm{Cu}, 154 \mathrm{mg} / \mathrm{kg}$ of $\mathrm{Pb}, 137 \mathrm{mg} / \mathrm{kg}$ of $\mathrm{Zn}, 8 \mathrm{mg} / \mathrm{kg}$ of $\mathrm{Ni}$, and $4.5(\mathrm{mg} / \mathrm{kg}) \mathrm{mg} / \mathrm{kg}$ of $\mathrm{Cr}$, respectively in samples A-E. The decreasing concentration order were as $\mathrm{Cu}>\mathrm{Pb}>\mathrm{Zn}>\mathrm{Ni}>\mathrm{Cr}$ for assessment level in 2015. The dumpsite soil and reference sample concentration indicate a clear difference between controlled and active sites for ewaste and non-e-waste activities. Furthermore, the average concentration of heavy metals for a single point with some statistical analysis is tabulated in (Table 1). The graphical representation is depicted in (Figure 1).

The detailed summarized comparison presented in table 2 from the year 2015-2018 with their respected acceptable values in soil by USEPA and Pak-EPA (Rafeeq et al., 2020)(Alam et al., 2015). Two elements $\mathrm{Fe}$ and $\mathrm{Cd}$ were added to the study. The overall concentrations for metal were found higher in 2015 than subsequent years, while in 2018 analysis was again increased than preceded two years. This may be the effect of low level of rain than the previous years. The order of metallic contamination was $\mathrm{Cu}>\mathrm{Zn}>\mathrm{Pb}>\mathrm{Fe}>\mathrm{Ni}>\mathrm{Cr}>$ $\mathrm{Cd}$. Maximum value for $\mathrm{Cu}, \mathrm{Zn}, \mathrm{Pb}, \mathrm{Fe} . \mathrm{Ni}, \mathrm{Cr}$ and Cd were observed as $332.41,125.35,111.95,5.90$, $5.74,2.91$ and $0.58 \mathrm{mg} / \mathrm{kg}$ respectively.

WD-XRF analysis was also carried out for the surface and depth soil samples in 2017 from the same dumpsite (shershah) Karachi. The maximum concentrations were found for $\mathrm{Cu}$ in sample $\mathrm{BS}, \mathrm{Zn}$ in $\mathrm{AS}, \mathrm{Pb}$ in $\mathrm{AS}, \mathrm{Fe}$ in $\mathrm{BS}, \mathrm{Ni}$ in $\mathrm{BS}$, and $\mathrm{Cr}$ in $\mathrm{AS}$, as $122.86,101.85,94.79,4.57,3.27$, and 0.82 $\mathrm{mg} / \mathrm{kg}$ respectively, while $\mathrm{Cd}$ was not detected in either for surface or depth composite samples shown in table 4 . The corresponding typical CRM was not confirmed as the instrument is purely installed for furnace refractory materials.

Another confirmation of the presence of metals in soil was carried out by SEM-imaging and SEMEDX for the samples collected in 2017. Fig 5, 8, 10, 13,16 were the images of samples AS, BS, AD, BD, and R. Fig $6 \& 7$ were showing in AS particle selection with its EDX. Fig 7 for BS, Fig $9 \& 10$ highlighting $\mathrm{AD}$ Fig 14 \& 15 representing BD, similarly reference sample connected with Fig 15. Maximum two images were confirming the focused metal in the soil as can be seen in EDX of the images. The morphological study confirmed the presence of metals in the soil. An evaluated concentration in $\mathrm{mg} / \mathrm{kg}$ on particle basis on different zooming power for the soil of surface and depth were presented in table 5 serial 1-8 with percentage absolute error at 1 sigma, and percentage relative error at 1 sigma.

The dust samples were also collected from dismantling sites in Karachi. The concentration of heavy metals such as $\mathrm{Cd}, \mathrm{Cr}, \mathrm{Cu}, \mathrm{Fe}, \mathrm{Ni}, \mathrm{Pb}$, and $\mathrm{Zn}$ was determined as given in Table 3 and graphically presented in Fig 2. The concentration of the metals was higher than the referenced sample values, indicating the pollutants difference in dismantling and control site, containing the nature of the samples. The $\mathrm{pH}$ of the samples was $7-8$, showing the alkaline nature of the samples.

Rubber from the toy (China toy) was analyzed for heavy metals using SEM-imaging \& SEM-EDX at $66 \mathrm{X}$. Three different points were considered to report which were showing the metallic presence. Figures 18, $19 \& 20$ are the images of these sample points with their EDXs. The metallic concentration in $\mathrm{mg} / \mathrm{kg}$ with percent absolute error at 1 sigma \& percent relative error at 1 sigma were tabulated in Table 6 . Although the results in $\mathrm{mg} / \mathrm{kg}$ were higher for selected metals but the absolute and relative error indicating the level of significance of the results, as lowest the error higher will be the accuracy. The fact is also that the SEM-EDX is not known for accurate quantitative analysis but for the qualitative study, SEM could be a primary tool (Zadora \& Brozek-Mucha, 2003) (Batista, Melo, Gilkes, \& Roberts, 2018).

Fig 3 is the image of surface soil of side A from which 2 particles at different magnification were selected to focus on required metals shown in Fig 4 at $277 \mathrm{X} \&$ Fig 5 at $463 \mathrm{X}$. Their EDXs provide the metallic concentration for $\mathrm{Cd}, \mathrm{Cr}, \mathrm{Cu}$, $\mathrm{Fe}, \mathrm{Ni}, \& \mathrm{~Pb}$ from Table 5 serial no 5 as 5842 , 10832, 7167, 45688, 778 \& $8747 \quad \mathrm{mg} / \mathrm{kg}$ respectively, while at $463 \mathrm{X}$ only four metals were detected having $\mathrm{Cr}, \mathrm{Cu}, \mathrm{Fe}, \& \mathrm{~Pb}$ with 563, 2927, $15011 \& 4043 \mathrm{mg} / \mathrm{kg}$ from Table 5 serial no 6 . At high magnification the Px $1.44 \mu \mathrm{m}$ was reduced to Px $0.86 \mu \mathrm{m}$ similarly a decline in concentration and 
absolute error is observed. On the other hand the relative error was increased. The trends of metals present in AS were observed as $\mathrm{Fe}>\mathrm{Cr}>\mathrm{Pb}>\mathrm{Cu}$ $>\mathrm{Cd}>\mathrm{Ni}$.

Side B surface sample image is shown in Fig 6. The required particle was found at $277 \mathrm{X}$, where Px was $1.44 \mu \mathrm{m}$. The detected metal concentrations shown were $65 \%$ less $\mathrm{Cd}, 95 \%$ low $\mathrm{Cr}, 88 \%$ higher $\mathrm{Cu}, 69 \%$ lower $\mathrm{Fe}, 66 \%$ elevated $\mathrm{Ni}$ and $86 \%$ lesser $\mathrm{Pb}$ content in $\mathrm{BS}$ as compared to AS (Table 5, serial no 7). The $\mathrm{Zn}$ was $3760 \mathrm{mg} / \mathrm{kg}$ in BS while $\mathrm{Zn}$ was not detected in AS. The results are showing that Bs is less contaminated for $\mathrm{Cd}, \mathrm{Fe}, \mathrm{Cr}$, and for $\mathrm{Pb}$ than AS.

SEM image was taken for side A depth sample and shown in Fig 8. The soil sample particle for AD at $92 \mathrm{X}$ gives the concentration for $\mathrm{Cd}, \mathrm{Cr}, \mathrm{Cu}, \mathrm{Fe}, \&$ $\mathrm{Ni}$ as $2809,376,3138,47126 \& 1055 \mathrm{mg} / \mathrm{kg}$ respectively from the Table 5 , which when further magnified at $185 \mathrm{X}$ it gives $\mathrm{Pb}$ and $\mathrm{Zn}$ shown in Fig 10 and analyzed by SEM-EDX spectrometer for the chemical composition of selected particle which was stated in table 5 , serial no $1 \& 2$. The results show the metallic trend as $\mathrm{Fe}>\mathrm{Pb}>\mathrm{Cu}>\mathrm{Zn}$ along their concentration as 14559, 3310, 1905 and 902 $\mathrm{mg} / \mathrm{kg}$ respectively.

Fig 11 shows the particles of side B depth soil sample image at $100 \mathrm{X}$ magnification power with SEM. Particle selected at 92X (Fig 12) was Px $4.33 \mu \mathrm{m}$ and analyzed on SEM-EDX for quantitative metallic component, shows the heavy metal concentration as chromium $(519 \mathrm{mg} / \mathrm{kg})$, Copper $(597 \mathrm{mg} / \mathrm{kg})$, Iron $(22113 \mathrm{mg} / \mathrm{kg})$, Nickel $(78 \mathrm{mg} / \mathrm{kg})$ \& Zinc $(1606 \mathrm{mg} / \mathrm{kg})$ with abs. Error at 1 sigma 370, $418,1345,52.8 \& 556 \mathrm{mg} / \mathrm{kg}$ while the relative error was $71.2,70.1,6.1,67.5 \& 34.7 \mathrm{mg} / \mathrm{kg}$ respectively from Table 5 serial no 3 . The increase in magnification power from $92 \mathrm{X}$ to $278 \mathrm{X}$ the SEMEDX shows the $\mathrm{Pb}$ (Fig 13) surprisingly $\mathrm{Fe} \& \mathrm{Ni}$ was disappeared. After zooming the metallic distribution order was $\mathrm{Pb}>\mathrm{Cu}>\mathrm{Cr}>\mathrm{Zn}$ and the EDX analyzer shows their concentration as 4239 , $2100,555 \& 367 \mathrm{mg} / \mathrm{kg}$ respectively presented in table 5 serial no 4 .

Non-e-waste site sample (R) particle was imaged by SEM shown in Fig 14 at 200X. After checking on a higher magnification level it was reverted to $185 \mathrm{X}$ as the EDX analysis shows only Iron $(\mathrm{Fe})$ content $35218 \mathrm{mg} / \mathrm{kg}$. A clear indication of non-contaminated soil present in the Nazimabad playground Karachi.

Figs 18-20 were the three best SEM images for the metallic particles present in the rubber sample at 66X and their relative EDXs. At point 1 the $\mathrm{Cr}, \mathrm{Fe}$, Ni \& Zn was detected with their concentration of $544,4735,186 \& 1298 \mathrm{mg} / \mathrm{kg}$ respectively (Fig 16, Table 6). Point 2 image and EDX evaluation depicted in Fig 17, Table 6, here $\mathrm{Cd}, \mathrm{Cr}, \mathrm{Fe} \& \mathrm{Ni}$ appears as the heavy metals while the EDX were conformed $755,322,5279 \& 580 \mathrm{mg} / \mathrm{kg}$ for their concentrations. Third point consideration provided $\mathrm{Pb}$ in addition to $\mathrm{Cd}, \mathrm{Cr}, \mathrm{Fe} \& \mathrm{Ni}$ with $983,47,430$, $7275 \& 407 \mathrm{mg} / \mathrm{kg}$ respectively (Fig 18, Table 6).

A comparison of the whole study has also been tabulated in Table 7. In which dumping site profiling, four-year detailed study, dismantling dust and rubber sample results were compared by their range values. Either the results were evaluated from AAS, WD-XRF, or SEM-EDX. All of the tabulated results are alarming for the e-waste activities as $\mathrm{Cu}$ $\& \mathrm{~Pb}$ are touching the maximum allowable limits of USEPA $300 \& 100 \mathrm{mg} / \mathrm{kg}$ for soil (Ofudje, 2014)(USEPA, 1997). Despite the mathematical expression and comparison with recommendations, the contamination level is the clear image as increased. Dismantling dust contamination directly impacts on the workers and the surrounding residential, as the lower level of $\mathrm{Cd}, 0.07 \mathrm{mg} / \mathrm{kg}$, and $\mathrm{Pb}, 10.56 \mathrm{mg} / \mathrm{kg}$ could directly pose a serious chronic threat to inhalers (USEPA, 1997). Rubber sample concentration for heavy metals are also threatened as the circle of contact for rubber or plastic is general public especially children from 0.5 - 6 years old, rather than the workers for e-waste. The metals in rubber also indicate the route map of EoL (end of life) product to recycled product (Dimitrakakis, Janz, Bilitewski, \& Gidarakos, 2009) (Vehlow et al., 2000) (Nnorom \& Osibanjo, 2009).

\section{CONCLUSION}

In this study, a complete analytical profile of heavy metals in soil, dismantling dust, and rubber (recycled product) was carried out in various samples collected from the electronic waste dumpsite and dismantling site of the Shershah market, and from the local market in Karachi. In the samples of soil, the higher average concentrations were found to be $373.87 \mathrm{mg} / \mathrm{kg}$ of $\mathrm{Cu}, 112.11$ $\mathrm{mg} / \mathrm{kg}$ of Pb, $125.41 \mathrm{mg} / \mathrm{kg}$ of $\mathrm{Zn}, 6.31 \mathrm{mg} / \mathrm{kg}$ of Ni 
and $2.44 \mathrm{mg} / \mathrm{kg}$ of $\mathrm{Cr}$, respectively. The dust samples were analyzed for heavy metals such as $\mathrm{Cd}$, $\mathrm{Cr}, \mathrm{Cu}, \mathrm{Fe}, \mathrm{Ni}, \mathrm{Pb}$, and $\mathrm{Zn}$ collected from dismantling sites. The metallic trend in dismantling dust was observed as $\mathrm{Zn}>\mathrm{Pb}>\mathrm{Fe}>\mathrm{Cu}>\mathrm{Cr}>\mathrm{Ni}>$ $\mathrm{Cd}$ with lead concentration of $10.56 \mathrm{mg} / \mathrm{kg}$ and $\mathrm{Cd}$ of $0.069 \mathrm{mg} / \mathrm{kg}$. Nearly the same results were also observed through WD-XRF for an alternate route of verification. The soil and rubber particle analysis by SEM-EDX was also indicating the presence of heavy metals. The concentration of the metals in soil and dust was higher than the controlled reference sample values, indicating the metallic pollutants containing the nature of the samples. The $\mathrm{pH}$ of samples was 7-8, showing the alkaline nature of the samples. This study will be valuable for selecting appropriate mitigation for the e-wasteimpacted deteriorated environments and to safeguard the environment and people from potential hazards. This quantitative analysis-based study would be helpful in future socio-economic indications if a deep medicated investigation is performed by anyone or any means.

\section{ACKNOWLEDGMENT}

Authors acknowledge financial support from the department of chemistry, University of Karachi, Karachi, Pakistan and People Steel mills Ltd, Karachi Pakistan for technical assistance.

\section{REFERENCES}

Adaramodu A. A., Osuntogun A. O., EhiEromosele C. O. (2012). Heavy Metal Concentration of Surface Dust Present in EWaste Components: The Westminister Electronic Market, Lagos Case Study. Resources and Environment, 2 (2), 9-13.

Ahmed, K., Hassan, N., Akhter, N., \& Mumtaz, M. (2019). Chemical and morphological characteristics of heavy and hazardous metals in settled dust associated with different functional areas of Karachi, Pakistan. 46 (3), 46-58.

Alam, N., Ahmad, S. R., Qadir, A., Ashraf, M. I., Lakhan, C., \& Lakhan, V. C. (2015). Use of statistical and GIS techniques to assess and predict concentrations of heavy metals in soils of Lahore City, Pakistan. Environmental Monitoring and Assessment, 187 (10).
Batista, A. H., Melo, V. F., Gilkes, R., \& Roberts, M. (2018). Identification of heavy metals in crystals of sand and silt fractions of soils by scanning electron microscopy (SEM EDS/WD-EPMA). Revista Brasileira de Ciencia Do Solo, 42, 1-16.

Bimir, M. N. (2020). Revisiting e-waste management practices in selected African countries. Journal of the Air and Waste Management Association, 70 (7), 659-669.

Dimitrakakis, E., Janz, A., Bilitewski, B., \& Gidarakos, E. (2009). Determination of heavy metals and halogens in plastics from electric and electronic waste. Waste Management, 29 (10), 2700-2706.

Ehi-Eromosele C.O, Adaramodu A.A, Anake W.U, Ajanaku, C. ., \& Edobor-Osoh, A. (2012). Comparison of Three Methods of Digestion for Trace Metal Analysis in Surface Dust Collected from an E-waste Recycling Site. Journal of Chemical Information and Modeling, 10 (10), 1689-1699.

Faheem Gul Gilal, Syed Mir Muhammad Shah, Sultan Adeel, Rukhsana Gul Gilal, N. G. G. (2021). Consumer e-waste disposal behaviour: A systematic review and research agenda. International Journal of Consumer Studies.

Forti, V., Baldé, C. P., Kuehr, R., \& Bel, G. (2020). The Global E-waste Monitor 2020. Retrieved from http://ewastemonitor.info/

Guo, Y., Huo, X., Li, Y., Wu, K., Liu, J., Huang, J., ... Xu, X. (2010). Monitoring of lead, cadmium, chromium and nickel in placenta from an e-waste recycling town in China. Science of the Total Environment, 408 (16), 3113-3117.

Hassan, N., \& Naseem, A. (2020). Study of Alkali Metal Oxides, Alkaline Earth Metal Oxides And Non-Metal Oxides In Settled Dust of Assorted Regions of Karachi, Pakistan Naveed Hassan, Naseem Akhter, Syed Sajid Hussain and Mohsin Mumtaz. (June).

Imran, M., Haydar, S., Kim, J., Awan, M. R., \& Bhatti, A. A. (2017). E-waste flows, resource recovery and improvement of legal framework in Pakistan. Resources, Conservation and Recycling, 125 (January), 131-138.

Iqbal, M., Breivik, K., Syed, J. H., Malik, R. N., Li, 
J., Zhang, G., \& Jones, K. C. (2015a). Emerging issue of e-waste in Pakistan: A review of status, research needs and data gaps. Environmental Pollution, 207, 308318.

Iqbal, M., Breivik, K., Syed, J. H., Malik, R. N., Li, J., Zhang, G., \& Jones, K. C. (2015b). Emerging issue of e-waste in Pakistan: A review of status, research needs and data gaps. Environmental Pollution, 207 (January), 308-318.

Iqbal, M., Syed, J. H., Breivik, K., Chaudhry, M. J. I., Li, J., Zhang, G., \& Malik, R. N. (2017). E-Waste Driven Pollution in Pakistan: The First Evidence of Environmental and Human Exposure to Flame Retardants (FRs) in Karachi City. Environmental Science and Technology, 51 (23), 13895-13905.

Islam, A., Ahmed, T., Awual, M. R., Rahman, A., Sultana, M., Aziz, A. A., ... Hasan, M. (2020). Advances in sustainable approaches to recover metals from e-waste-A review. Journal of Cleaner Production, 244, 118815.

Juneng, L., Latif, M. T., \& Tangang, F. (2011). Factors influencing the variations of PM10 aerosol dust in Klang Valley, Malaysia during the summer. Atmospheric Environment, 45 (26), 4370-4378.

Kalamaras, G., Kloukinioti, M., Antonopoulou, M., Ntaikou, I., Vlastos, D., Eleftherianos, A., \& Dailianis, S. (2021). The potential risk of electronic waste disposal into aquatic media: The case of personal computer motherboards. Toxics, 9 (7).

Leung, Anna O. W. et al., 2008. (2008). Heavy Metals Concentrations of Surface Dust from e-Waste Recycling and Its Human Health Implications in Southeast China. Environ. Sci. Technol, 42 (7), 2674.

Li, W., \& Achal, V. (2020). Environmental and health impacts due to e-waste disposal in China - A review. Science of the Total Environment, 737, 139745.

Liu, Y., Huo, X., Xu, L., Wei, X., Wu, W., Wu, X., \& Xu, X. (2018). Hearing loss in children with e-waste lead and cadmium exposure. Science of the Total Environment, 624, 621627.

Michelle, H., Bain, K. C., Ansong, A. K., S., B. L., Lennart, B. Å., Marie-Noel, B., ... A., S. W.
(2016). E-Waste and Harm to Vulnerable Populations: A Growing Global Problem. Environmental Health Perspectives, 124 (5), 550-555.

Mostafaii, G. R., Bakhtyari, Z., Atoof, F., Baziar, M., Fouladi-Fard, R., Rezaali, M., \& Mirzaei, N. (2021). Health risk assessment and source apportionment of heavy metals in atmospheric dustfall in a city of Khuzestan Province, Iran. Journal of Environmental Health Science and Engineering. https://doi.org/10.1007/s40201-021-00630-z

Nnorom, I. C., \& Osibanjo, O. (2009). Toxicity characterization of waste mobile phone plastics. Journal of Hazardous Materials, 161 (1), 183-188.

Ofudje, E. (2014). Heavy Metals Concentration at Electronic-Waste Dismantling Sites and Dumpsites in Lagos, Nigeria. International Research Journal of Pure and Applied Chemistry, 4 (6), 678-690.

Ohajinwa, C. M., van Bodegom, P. M., Vijver, M. G., \& Peijnenburg, W. J. G. M. (2018). Impact of informal electronic waste recycling on metal concentrations in soils and dusts. Environmental Research, 164 (December 2017), 385-394.

Olafisoye, O. B., Adefioye, T., \& Osibote, O. A. (2013). Heavy metals contamination of water, soil, and plants around an electronic waste dumpsite. Polish Journal of Environmental Studies, 22 (5), 1431-1439.

Patil, R. A., \& Ramakrishna, S. (2020). A comprehensive analysis of e-waste legislation worldwide. Environmental Science and Pollution Research, 27 (13), 14412-14431.

Perkins, D. N., Brune Drisse, M. N., Nxele, T., \& Sly, P. D. (2014). E-waste: A global hazard. Annals of Global Health, 80(4), 286-295. https://doi.org/10.1016/j.aogh.2014.10.001

Rafeeq, A. (2019). Determination of Toxic Metals from Karachi Region Electronic Waste Dumpsite in (karachi). Retrieved from Submitted to HEC Pakistan

Rafeeq, A., Ali, S. A., Tanoli, A. K., Shah, A. R., Khan, A., \& Mumtaz, M. (2020). Soil contamination due to heavy metals at electronic waste dumpsites in Karachi, Pakistan. Pakistan Journal of Analytical and 
Environmental Chemistry, 21 (2), 332-341.

Shaikh, S., Thomas, K., Zuhair, S., \& Magalini, F. (2020). A cost-benefit analysis of the downstream impacts of e-waste recycling in Pakistan. Waste Management, 118.

Singh, N., Duan, H., Ogunseitan, O. A., Li, J., \& Tang, Y. (2019). Toxicity trends in E-Waste: A comparative analysis of metals in discarded mobile phones. Journal of Hazardous Materials, 380 (July), 120898.

Singh, N., Duan, H., \& Tang, Y. (2020). Toxicity evaluation of E-waste plastics and potential repercussions for human health. Environment International, 137(September 2019), 105559.

Umair, S., Anderberg, S., \& Potting, J. (2016). Informal Electronic Waste Recycling in Pakistan. The Journal of Solid Waste Technology and Management, 42 (3), 222235.

Umair, S., Björklund, A., \& Petersen, E. E. (2015). Social impact assessment of informal recycling of electronic ICT waste in Pakistan using UNEP SETAC guidelines. Resources, Conservation and Recycling, 95, 46-57.

USEPA. (1997). Update to Exposure Factors Handbook. Environmental Protection, I (August).

Vehlow, J., Bergfeldt, B., Jay, K., Seifert, H., Wanke, T., \& Mark, F. E. (2000). Thermal treatment of electrical and electronic waste plastics. Waste Management \& Research, 18 (2), 131-140.

Xu, X., Zeng, X., Boezen, H. M., \& Huo, X. (2015). E-waste environmental contamination and harm to public health in China. Frontiers of Medicine, 9 (2), 220-228.

Zadora, G., \& Brozek-Mucha, Z. (2003). SEMEDX - A useful tool for forensic examinations. Materials Chemistry and Physics, 81 (2-3), 345-348.

Zeng, X., Xu, X., Boezen, H. M., \& Huo, X. (2016). Children with health impairments by heavy metals in an e-waste recycling area. Chemosphere, 148, 408-415. 